Itinéraires Itinéraires

Littérature, textes, cultures

2011-2 | 2011

Le concept de mémoire

\title{
Hugues de Chanay et Odile Le Guern (dir.), Signes du corps, corps du signe
}

Paris, L'Harmattan, 2009

\section{Marina Krylyschin et Catherine Ruchon}

\section{(2) OpenEdition}

\section{Journals}

Édition électronique

URL : http://journals.openedition.org/itineraires/191

DOI : 10.4000/itineraires. 191

ISSN : 2427-920X

Éditeur

Pléiade

Édition imprimée

Date de publication : 1 juillet 2011

Pagination : 175-180

ISBN : 978-2-296-54673-8

ISSN : 2100-1340

Référence électronique

Marina Krylyschin et Catherine Ruchon, « Hugues de Chanay et Odile Le Guern (dir.), Signes du corps, corps du signe », Itinéraires [En ligne], 2011-2 | 2011, mis en ligne le 13 janvier 2014, consulté le 22 septembre 2020. URL : http://journals.openedition.org/itineraires/191 ; DOI : https://doi.org/10.4000/ itineraires. 191 


\section{Comptes rendus}

Hugues de Chanay et Odile Le Guern (dir.), Signes $d u$ corps, corps $d u$ signe, Paris, L'Harmattan, 2009, 282 p. ISBN : 978-2-296-08219-9.

Cet ouvrage rassemble les diverses communications de la session préparatoire en septembre 2002 du huitième congrès de l'Association Internationale de Sémiotique qui eut lieu deux ans plus tard à Lyon.

Signes du corps, corps du signe nous raconte l'entrelacement de relations subtiles entre le symbole et le réel. Il donne un éclairage à multiples facettes sur un présupposé trop souvent mis à l'écart, celui du signifiant, de la matérialité du signe, et remet aussi en lumière celui qui se trouve à la source du signe. Les nombreuses réalités que recouvre le signe dans les domaines de l'art, de la poésie, de la littérature, de la danse, du théâtre, de la religion, etc., constituent la force et la richesse de cet ouvrage collectif.

La première partie de l'ouvrage propose de circonscrire le champ théorique de la matérialité du signe en regroupant plusieurs approches.

Quand la matérialité du signe se prolonge dans les sensations qu'il éveille chez l'énonciateur ou l'énonciataire, on peut alors, comme Roland Posner et Dagmar Schmauks (Berlin) dans « Synaesthesia : Physiological Diagnosis, Practice of Perception, Art Program. A semiotic re-analysis », parler de synesthésie, perception simultanée relevant de différentes modalités sensorielles. Cette pathologie s'expérimente de façon plus ordinaire dans des expressions langagières (une musique colorée), dans l'art (qui exprime la même idée sous différentes matérialités) ou la religion. L'histoire des perceptions montre comment les sens ont été marginalisés, placés sous la maîtrise de l'intellect, anesthésiés, déconnectés les uns des autres et de la réalité. Par l'absence même du stimulus, la synesthésie permet la métaphore, la symbolisation, la sublimation.

Dans «L'aube du langage : la médiation esthétique du signe », Bernard Lamizet (Lyon), nous montre que l'expérience esthétique est une régression vers l'aube du langage, vers les formes a priori de la communication, de 
l'échange symbolique. Par les formes et les représentations (poésie, danse, etc.), le corps exprime une « signification interprétée du sujet». Il y a médiation esthétique du signe par le corps. Le corps se fait signe, autorisant une reconnaissance, une interprétation. Alors, le sujet est confronté à la matérialité interprétable de ce qui fait signe, à l'évidence du corps du signe. Cette expérience (où l'autre n'a pas de place) permet de redécouvrir la matérialité originaire du signe, un état avant même que le signe ne signifie.

À cette vision sensorielle de la matérialité du signe répond l'approche matérialiste et économique de Ferruccio Rossi-Landi présentée par Jeff Bernard (Vienne) dans « Co-presence of Artefacts ». L'auteur y déploie les concepts clés de la théorie de F. Rossi-Landi comme le travail du signe, l'homologie entre production matérielle et production du signe, la reproduction sociale. J. Bernard propose d'élargir ce modèle socio-sémiotique par l'ajout aux artefacts matériels des signifacts (signes de communication externes) et des mentefacts (signes idéologiques internes). La sémiosis est le résultat d'un travail qui produit un signe formé de différentes parties dont il persiste des résidus détectables par l'historicité et de la socialité.

Dans « Bodies and Signs : For a Typology of Semiosic Materiality », Susan Petrilli (Italie) apporte une dimension éthique à cette réflexion sur la matérialité du signe en élargissant l'étude au-delà du monde humain et en réintégrant le biologique. Matérialité dont elle dresse une typologie hiérarchisée, s'appuyant elle aussi sur Ferruccio Rossi-Landi, mais encore sur Thomas A. Sebeok et Charles S. Peirce. Dans un monde modalisé par le langage (et ici sémiotique s'oppose à sémiosis), la matérialité sémiotique engage pleinement la responsabilité humaine envers la vie sur terre : seul l'être humain a cette capacité sémiotique, et en tant que tel a le devoir de veiller au vivant.

Dans « Corps, langage et altérité chez Emmanuel Lévinas », Augusto Ponzio (Italie) replace la discussion sur le plan éthique amorcée par Susan Petrilli en posant la question de l'altérité chez Emmanuel Lévinas. L'identité, le soi, la séparation d'avec l'autre, se construisent dans l'altérité (par le biais de la jouissance). La fonction présentative (phatique et vocative) du langage montre le désir que l'autre soit présent. Cette non-indifférence pour l'autre relève de l'éthique telle que la conçoit Lévinas. C'est le corps qui expose le soi à l'altérité et permet l'intercorporéité avec tous les vivants, rejoignant en cela la sémio-éthique de Pétrilli et Ponzio. La substitution aux autres, cette « altérité dans le même », ne lèse en rien toutefois l'unicité du je.

Avec Solomon Marcus, nous entrons dans le domaine du vivant. Dans « The Body Identity : From Material and Structural to Global, Semiotic and Computational », l'auteur développe une typologie identitaire du corps humain : l'identité matérielle (niveau macroscopique), structurelle (niveau infinitésimal reposant sur la disposition des cellules), " computationnelle » (ce que le corps peut faire), sémiotique (ce qu'il signifie) et le champ identitaire (défini par l'interaction permanente avec le monde extérieur). 
Le champ identitaire est une notion capitale dans le contexte d'une mondialisation souvent perçue comme une menace pour notre identité.

À l'instar de Susan Petrilli, John Deely (Houston) définit l'être humain par sa capacité sémiotique. Dans « Il ne suffit pas d'un corps pour faire un signe », son approche du signe est philosophique. Selon la définition de T. A. Sebeok, l'animal est incapable de différencier l'objet (concret) de la chose (abstraite). Tandis que l'homme parlant, capable d'abstraction, n'est plus une «res cogitans » mais un animal sémiotique car le seul à voir ce qui unit les choses reliées. C'est ainsi que l'on peut intercaler entre les deux faces du signe saussurien un troisième élément, celui de la relation.

Dinda Gorlée (Helsinki) confronte la pensée de deux éminents sémiologues, celle du structuraliste français Roland Barthes et celle du pragmaticien britannique C. S. Peirce. On peut voir une convergence entre certaines positions de Barthes sur le texte et les concepts de priméité, secondéité et tiercéité de Peirce. Priméité et plaisir du texte, secondéité et études épistémologiques du texte... Cependant, la logique objective et le pragmatisme de Peirce se heurtent à la conception barthésienne d'une réalité et d'une signification insaisissables. En dépit des apparences, ces deux penseurs incarnent deux théories sémiotiques opposées.

La seconde partie de l'ouvrage propose d'étudier les différentes réalités que recouvre le signe à travers des études de corpus émanant de différents genres discursifs (biblique, touristique, littéraire, publicitaire) ainsi que de corpus polysémiotiques (audiovisuels, picturaux, cinématographiques, lyriques, architecturaux).

Dans « Le signe rompu du corps : modèles sémiotiques et discours théologiques », Louis Panier (Lyon) explore les conditions sémiotiques de l'énoncé « Ceci est mon corps donné pour vous ». La modalité assertive soutient une métaphore entre le pain et le corps de l'énonciateur. Cependant l'auteur dépasse l'interprétation purement symbolique, où le « pain » serait signifiant et le « corps » signifié. La parole sur le pain manifeste la coupure de l'énonciation (schieze) qui rend la sémiose possible par un acte de détachement (« ceci » est « mon » corps); cette division fait du corps le lieu de la parole. Ce dispositif de division, cette brèche ouverte, permet la répétition des figures (" ceci est mon sang ») dans l'attente d'un interprétant final, d'un corps pour le sujet de l'énonciation.

Dans « Le délit du corps », Marie C. Poix-Têtu analyse la représentation d'une mère mourante en mettant en parallèle deux lectures de Marguerite Yourcenar : Anna, Soror... et « Le lait de la mort » avec un extrait de Souvenirs pieux. Selon les versions d'Anna, Soror..., la représentation de la mort - et des signes du corps affecté par elle - peut disparaître. Toutefois, des traces de cette évocation du corps au bord du cadre, qu'est le délit du corps, ont été retrouvées, reprises et déposées dans Souvenirs pieux. 
L'auteur fait l'hypothèse que, par ce qu'elles persistent à taire ou à dire d'une version à l'autre, les variantes laissent apparaître, en filigrane, toute la chair d'un texte, que parfois personne ne parvient jamais à énoncer.

Dans « Le corps du voyageur dans la description géographique », Pierre Sadoulet compare la représentation du corps à travers un guide de randonnées pédestre et les récits de voyageurs. Il montre que ces discours de représentation de l'espace reposent sur un processus de tension/refoulement : un refoulement du corps et de ses manifestations (fatigue, souffrance, angoisse face aux dangers et possibilités d'égarement) et la présence d'un corps tensif présupposé (lexique du déplacement, importance des « points de vue »). Mais le corps refoulé n'est pas un corps absent et le plaisir du randonneur est à son comble lorsqu'il trouve une cohérence entre la phénoménologie de la marche et la rationalité de la carte.

Dans « Figures du corps et énonciation », Nathalie Simon propose deux études qui abordent la question référentielle du corps à partir de son inscription figurative dans deux discours, l'un littéraire, l'autre publicitaire, avec l'idée de montrer comment, plus que la référentiation, l'énonciation, qui est à l'œuvre dans les discours, influence l'énonciataire. L'auteure montre ainsi comment l'impression référentielle d'un corps découle davantage de l'énonciation des qualités sensibles, graphiques et chromatique, d'une robe qui l'enveloppe et l'iconise... ou le déréalise et le désiconise.

Adrian Gimate-Welsh et Maria Rayo Sankey Garcia proposent dans " Gestures and the Notion of Democracy » une analyse " paralinguistique » des discours en étudiant la gestuelle de six personnalités politiques mexicaines lors de débats télévisés de trois minutes chacun. Pour les auteurs, les gestes des mains, droite ou gauche, combinés aux hochements de tête, constituent à la fois une sorte de lexique et une syntaxe de la gestuelle. Ils constituent une fenêtre sur l'esprit de l'énonciateur et sont le signe d'une interaction avec autrui. L'hypothèse est que les gestes en politique sont le reflet d'une « cartographie cognitive » différente selon l'ancrage idéologique des locuteurs. Ainsi les gestes, avec leur façon d'occuper et de découper l'espace, seraient les indices révélateurs des différentes conceptions qu'auraient les énonciateurs de la démocratie.

Dans «Cosmos, Body and Aesthetic Urban Space », Alexandros $\mathrm{Ph}$. Lagopoulos nous rappelle, à travers les exemples du village Fali du kangu (au Nord Cameroun) et de mandalas tibétains, comment le corps et le code anthropomorphique, étroitement liés au code cosmique, constituent une dimension symbolique majeure de l'espace. Dans les sociétés occidentales, les proportions (mathématique et symétrique) du corps humain sont devenues au fil des siècles les « mesures étalon » à partir desquelles l'espace est organisé (Vitruve, Alberti, Le Corbusier). Certaines métaphores restituent aujourd'hui encore cette relation intime entre le corps et l'espace urbanisé comme les artères, le cœur, ou le poumon de la ville pour désigner un espace vert. 
Dans « La gestualité dans la visualisation du texte biblique », Vassela Posner analyse, à travers les images religieuses, l'utilisation de la corporalité en tant que système de signes visuels dans des cultures chrétiennes de l'Est (orthodoxe) et de l'Ouest (catholique et protestante). L'auteure propose une approche pragmatique des gestes, qui peuvent être interprétés comme des images aux propriétés performatives - prescriptives ou promissives - agissant sur la réalité du spectateur et dont le contenu sémantique, codifié, varie selon l'histoire du christianisme et les pratiques gestuelles dans les différentes cultures.

Dans son article «L'autoportrait, signe du corps par le corps du signe », Odile Le Guern analyse le double mouvement du peintre qui, à travers son autoportrait, se révèle et se dérobe au regard de l'observateur. Dans l'autoportrait, signe du corps de l'artiste, ce dernier, en tant que sujet énonçant, s'y révèle le plus souvent par la représentation iconique d'attributs (pinceaux, palettes) qui constituent une façon de parler de l'énonciation picturale, mais c'est dans le travail de la matière picturale, considérée comme le corps du signe qu'est l'autoportrait, que le peintre se dévoile. Dans ce travail de déformation, de réécriture, de métamorphose de son visage, le peintre construit « un discours sur soi ». Ce conflit entre iconique et pictural, contenu et expression, entre apparence et apparaître, permet ainsi à l'artiste énonciateur, de s'exposer tout en se dérobant.

Dans « On the Materiality of Film : The Diegetization of Stylistic Devices », Gloria Withalm étudie l' « auto-réflexivité » à l'œuvre dans certains films, c'est-à-dire la manière dont le film « parle » de sa mise en film, en laissant apparaître des marques d'énonciation, comme le recours au noir et blanc dans une même œuvre, ou de fabrication (générique de début, de fin, sous-titres, insertion de graphismes ou de textes dans l'image (Tex Avery), intégration dans des dialogues d'informations relatives aux aspects techniques du film, référence au caractère périssable du film en tant que support (Cinéma paradisio de Guiseppe Tornatore). L'auteure montre ainsi comment des éléments extra-diégétiques entrent parfois dans l'espace diégétique de la fiction.

Dans « La chair des sons, ou l'aspectualisation du corps : l'exemple de l'opéra », Hugues de Chanay analyse la « justesse » de la sensation que l'on éprouve à l'écoute d'une pièce lyrique. Ce trouble esthétique est le fruit des effets de voix, la chair des sons, qui transmettent l'émotion, permettant ainsi la catharsis qui repose elle-même sur une double empathie, l'une narrative (le livret, le sens), l'autre somatique (respiration, rythme, intensité...). Ces différentes formes d'empathie sont mises en jeu dans l'aspectualisation, qui réside dans l'ajustement du dire sur le vécu, par le jeu de débordements ou d'immersion des vocalises. L'adéquation entre sens et sensation, à l'origine du sentiment de « justesse », est ce que l'on cherche à rééprouver, au fil des écoutes répétées d'un même morceau.

La perspective pluridisciplinaire de cet ouvrage et la diversité des objets d'analyse exposés font apparaître la dynamique du signe et de ses 
relations intrinsèques (avec les signifiants, les énonciateurs et les énonciataires) et illustrent bien l'idée selon laquelle le signe fait émerger le corps, le corps fait émerger le signe, et que la signifiance naît de cette réciprocité.

\author{
Marina Krylyschin \\ Université de Paris $5-E D A$ \\ Catherine Ruchon \\ Université Paris 13 - CENEL
}

Alice Krieg-Planque, La Notion de «formule » en analyse du discours. Cadre théorique et méthodologique, Besançon, Presses universitaires de Franche-Comté, 2009, 144 p. ISBN : 978-2-84867-255-7.

Cet ouvrage d'Alice Krieg-Planque fait suite à un premier travail initié en thèse de doctorat en 2000 sur Émergence et emplois de la formule "purification ethnique " dans la presse française (1980-1994) et dont la présentation des résultats et leur analyse en contexte a fait l'objet, trois ans plus tard, d'une publication remaniée aux éditions du CNRS ${ }^{1}$. Elle s'y propose fondamentalement un dessein plus élaboré qui donne une meilleure visibilité au cadre théorique (l' « école française d'analyse du discours ») et méthodologique (détermination des contours de l'objet dans le cadre d'une approche pluridisciplinaire) qui éclaire son analyse de la notion de formule définie comme « un ensemble de formulations qui, du fait de leur emploi à un moment donné et dans un espace public donné, cristallisent des enjeux politiques et sociaux que ces expressions contribuent dans le même temps à construire » (p. 7). Mais au-delà de cette notion de formule, l'ouvrage explore aussi d'autres phénomènes de reprise discursifs comme les « petites phrases » ou les « slogans » qui, tous, permettent de s'apercevoir des rapports interactionnels d'opinion, de domination, etc. qui peuvent exister entre les différents acteurs d'une même sphère socio-politique.

Sur le plan de la structure et de l'architecture, le texte s'organise en cinq chapitres qui interrogent tour à tour et comme suit : des travaux sélectifs sur les usages socio-politiques de mots qui permettent de repérer des formules (chapitre I), le travail heuristique opéré par Jean-Pierre Faye sur la formule État total qui, au-delà de la genèse qu'elle fait du terme de formule même, définit sa modalité de circulation et son caractère constitutivement figé (chapitre II), l'analyse de Marianne Ebel et Pierre Fiala

1. "Purification ethnique ". Une formule et son histoire, Paris, CNRS Éditions, coll. «Communication », 2003, 523 p. 